\title{
Inovação em um Sistema de Informação para Gestão de Acidentes de Trânsito na Perspectiva de Produto e Processo
}

\author{
Rogério Luis Rizzi ${ }^{1}$, Claudia Brandelero Rizzi ${ }^{1}$, Ivonei Freitas da Silva ${ }^{1}$, Frank P. \\ Tominc ${ }^{1}$
}

${ }^{1}$ Centro de Ciências Exatas e Tecnológicas (CCET) - Universidade Estadual do Oeste do Paraná (UNIOESTE)

Rua Universitária, 2069, Bairro: Jardim Universitário, CEP 85819-110, Cascavel Paraná- Brasil

\{rogeriorizzi,claudia_rizzi\}@hotmail.com, ivonei.silva@unioeste.br, frankptominc@gmail.com

\begin{abstract}
This paper presents a Geographic Information System, GIS for decision making support, concerning traffic accidents and their victims at municipal level and innovative aspects in the process of development of the system are discussed, emphasizing their design and technologies used in its implementation, and innovative aspects in the product by means of the EPP Methodology that adopts and incorporates. Comments are made on the previous version of the SIG, monolithic, and the proposal to microservices oriented architecture version. Motivations and challenges related to the use of functional programming paradigm and microservices are emphasized. These features distinguish the GIS of the other solutions in the same segment of application.
\end{abstract}

Resumo. Neste trabalho apresenta-se um Sistema de Informações Geográfico, SIG, para apoio à tomada de decisão de acidentes e acidentados de trânsito em âmbito municipal e são discutidos aspectos inovadores no seu processo de desenvolvimento, enfatizando sua concepção e as tecnologias utilizadas em sua implementação, bem como aspectos inovadores no produto por meio da metodologia que adota e incorpora. São feitos comentários sobre a versão anterior do SIG, monolítica, e a proposta para a versão orientada à arquitetura de microserviços. Motivações e desafios relativos ao uso de paradigma de programação funcional e microserviços são apontados. Essas características distinguem o SIG de outras soluções computacionais no mesmo segmento de aplicação.

\section{Introdução}

Acidentes de trânsito são eventos danosos que envolvem pessoas, veículos, ambiente e instituições, e para serem assim caracterizados, é necessária a presença de pelo menos dois desses fatores [Transitobr 2016]. Os desdobramentos às pessoas, decorrentes de acidentes, referem-se ao translado do acidentado ou morto; cuidados em saúde envolvendo as fases pré-hospitalar, hospitalar e pós-hospitalar; questões previdenciárias em função da impossibilidade de trabalhar, temporária ou permanente; perda de produção resultante da interrupção de atividades produtivas. Em relação aos veículos estão seus danos materiais; perda de carga; guincho ou remoção; dentre outros. Quanto

RIZZI, R. L.; RIZZI, C. B.; SILVA, I. F.; TOMINC, F. P.

Inovação em um Sistema de Informação para Gestão de Acidentes de Trânsito na Perspectiva de Produto e Processo

isys | Revista Brasileira de Sistemas de Informação, Rio de Janeiro, vol. 9, No. 3, pp. 05-25, 2016 
ao ambiente, estão inclusos os danos à propriedade pública e privada, e quanto às instituições estão os custos associados à estrutura judicial para o atendimento às questões referentes aos acidentes de trânsito [IPEAa 2006].

Em relatório de pesquisa recente, o Instituto de Pesquisa Econômica Aplicada, IPEA, divulgou os custos associados aos acidentes ocorridos nas rodovias federais brasileiras, em 2014. Os 170 mil acidentes de trânsito geraram um custo de R\$12,3 bilhões, e destes, 64,7\% foram associados às vítimas dos acidentes, considerando cuidados com a saúde e perda de produção devido às lesões ou morte; 34,7\% foram associados aos veículos, referentes aos danos materiais, perda de cargas e remoção dos veículos acidentados [IPEA 2015]. Essas informações apenas ilustram a dimensão do problema, visto se referir exclusivamente às rodovias federais desconsiderando os acidentes nas malhas viárias estaduais e municipais.

Acidentes de trânsito acarretam consequências físicas e psicológicas que repercutem sobre a vida dos acidentados e de seus familiares e de outros indivíduos que tenham convivido com a problemática [Mesquita Filho 2012]. Embora todos esses aspectos, muitos dos acidentes são evitáveis e passíveis de prevenção, o que torna ainda mais importante à realização de estudos e execução de ações que possam mitigá-los.

Estudos específicos no contexto de acidentes de trânsito enfrentam o obstáculo da não disponibilidade de dados estatísticos oficiais atualizados e completos. Dados relativos à 2014 mostram que ocorreram 43.075 óbitos e 201.000 feridos hospitalizados. Informação do Seguro de Danos Pessoais Causados por Veículos Automotores de Vias Terrestres, DPVAT, indicou que em 2014 ocorreram 52.200 indenizações por morte e 596.000 por invalidez [Por Vias Seguras 2016].

Motivos à defasagem de informações estão elencados no documento "Proposta para o Brasil para Redução de acidentes e Segurança Viária" [Brasil 2009]. Ele reconhece que os dados relativos aos acidentes de trânsito com destaque às mortes no país, são questionáveis, devido a não existência de um Sistema de Informação Nacional que os integre e que possa fornecer estatísticas confiáveis e completas. Um Sistema Nacional forneceria informações sobre o acidente, e a identificação da gravidade das lesões, tratamentos, intervenções realizadas e o acompanhamento às vítimas.

$\mathrm{Na}$ indisponibilidade de tais dados, responsáveis e gestores públicos geralmente tomam medidas de prevenção e promoção à saúde e ao trânsito de maneira empírica. A disponibilidade de um Sistema de Informações Georreferenciado, SIG, voltado à gestão e à tomada de decisão, pode contribuir para mitigar este cenário, viabilizando ações fundamentadas à redução de acidentes por meio de intervenções qualificadas e pontuais.

A inexistência de um SIG que contemple na íntegra tais características é motivação e justificativa à concepção do Sistema que aqui é apresentado, destacando-se nele os seus aspectos de inovação de produto e de processo de desenvolvimento. Com relação ao produto, ele incorpora a Metodologia de Estratégia de Proatividade e Parceria (EPP) divulgada pelo Global Road Safety Partnership (GRSP) [GRSP 2016], sintetizada na seção 3.1. Quanto ao processo de desenvolvimento, ele é realizado por meio da adoção de arquitetura e paradigma de programação que atendem aos atributos de qualidade, como a manutenibilidade e a minimização da quantidade de erros no 
código do software construído. Sua arquitetura é estruturada por meio de microserviços web implementados com paradigma funcional.

Assim sendo, o objetivo deste trabalho é apresentar um SIG para gestão de informações relativas aos acidentes e acidentados de trânsito, e discutir aspectos inovadores em termos de produto, viabilizados pela metodologia EPP embutida nas funcionalidades disponibilizadas, e em termos do processo de desenvolvimento do Sistema, enfatizando sua arquitetura e tecnologias utilizadas.

Para isso, na seção 2 são feitos discussões sobre como ocorrem, geralmente, nos municípios o registro de acidentes de trânsito; é feita também a apresentação de alguns sistemas em âmbito nacional e internacional e a apresentação do SIGETRANS (Sistema Integrado Georreferenciado de Controle e Monitoramento de Acidentes de Trânsito). Na seção 3 são conceituadas e pontuadas as questões de inovação relativas ao Sistema, enfatizando a inovação no produto e no paradigma de programação, arquitetura e tecnologias. Essas questões são retomadas, comparando a versão anterior do Sistema, monolítica, com a versão atual voltada a microserviços, assim como as lições aprendidas e os desafios enfrentados. Por fim, na seção 4 são feitas considerações finais.

\section{Registros de Acidentes de Trânsito em Cascavel-PR e Sistemas de Gestão}

Cascavel, como outras cidades brasileiras, enfrenta o problema relativo ao registro de acidentes de trânsito. Eles eram realizados pelas entidades municipais atuantes na área como Corpo de Bombeiros, Polícias Estadual e Federal, Companhia Municipal de Engenharia de Transporte e Trânsito e Concessionárias de Rodovias. Relativamente às vítimas, efetivavam os registros os Hospitais e Unidades de Pronto Atendimento, sendo que, algumas dessas entidades realizam o registro manualmente em formulários físicos, que nem sempre estavam completos, podendo estar replicados o que não viabilizava o relacionamento entre o acidente e o acompanhamento às suas vítimas.

Tais entidades, quando informatizadas, usam diferentes e não integrados Sistemas de Informações, que em decorrência, fornecem informações e estatísticas imprecisas e que não refletem a realidade do problema, dificultando a adoção de medidas preventivas e corretivas aos acidentes e acidentados. Exemplos são a falta de identificação dos locais onde ocorrem os acidentes, a não identificação de quais são os principais tipos de acidentes, ou as características das vias.

Outro grande problema é a ausência de informações adequadas sobre as vítimas dos acidentes, cujo os dados são colhidos e armazenados nas bases de dados dos hospitais e entidades públicas de saúde responsáveis pelo atendimento e pela estrutura de sua organização, dificultando, então, a completa identificação dos envolvidos e das vítimas. Como consequência, o poder público municipal não conhecia quais eram as principais lesões, os procedimentos emergenciais e hospitalares realizados, nem acompanhava as consequências sociais, físicas, psicológicas e econômicas dos sequelados e de seus familiares.

Objetivando superar essas dificuldades, principalmente por meio da constituição e articulação dos integrantes do Comitê Intersetorial de Prevenção e Controle de 
Acidentes de Trânsito do Município de Cascavel (COTRANS) ${ }^{1}$, os diferentes Setores que atuam no município, tanto na assistência inicial aos acidentes (Corpo de Bombeiros, Polícias Rodoviárias, e outros) bem como na assistência às vítimas (Hospitais, Unidades de Saúde e outros) entenderam a importância de integrar os dados em um único repositório, o Sistema SIGETRANS.

Porém, antes de apresentar o Sistema, para bem caracterizá-lo são realizados comentários a respeito de Sistemas de Informações que tratam de dados relativos ao trânsito. Embora eles não contemplem ocorrências com e sem vítimas, e não adotam uma metodologia específica à gestão de acidentes e vítimas como o SIGETRANS, eles são relevantes aos vários propósitos que se destinam.

Um deles é o Urban Traffic Management and Control (UTMC), que dispõe de diversas aplicações voltadas à gestão de tráfego, como o controle de tráfego urbano; transporte público em tempo real e informações de trânsito; medidas de aplicação de monitoramento; detecção de incidentes; informações de estacionamento; prioridade no transporte público; controle de poluição. Outra característica do Sistema é que os dados de diferentes aplicações podem ser organizados em um único banco de dados com dois propósitos: fornecer ferramentas de gestão e informar tendências e relações entre o congestionamento, a poluição, os níveis de tráfego e o clima [Babtie 1999].

Outro Sistema em âmbito internacional é o Split, Cycle and Offset Optimisation Technique (SCOOT1), que objetiva contribuir à gestão e controle de tráfego, e à redução de congestionamentos. Dentre suas características estão à priorização do transporte público; a detecção de incidentes e informações de trânsito. O Sistema, seu funcionamento, as versões existentes, as informações disponibilizadas, dentre outros aspectos são apresentados em [SCOOT 2012].

Outro Sistema é o da IBM que contém um módulo de gestão inteligente de transporte compartilhado com outros departamentos da cidade. Ele é descrito no documento produzido por [Cotton 2015], e objetiva fornecer aos gestores uma visibilidade da cidade e da rede de transporte para melhorar a resposta aos incidentes, e busca contribuir à avaliação de padrões de tráfego e prever as chegadas de transporte público para melhorar o gerenciamento dos congestionamentos e a satisfação de passageiros e transportadores.

Em âmbito nacional, o Sistema de Informações de Acidentes de Trânsito (SAT) do Distrito Federal visa subsidiar o planejamento e a avaliação de ações voltadas à redução de acidentes de trânsito com vitimas [SAT 2016]. Já o Sistema de Informações Gerenciais de Acidentes de Trânsito do Estado de São Paulo, (InfoSiga), consiste em um banco de dados referentes aos acidentes de trânsito ocorridos no Estado. O Sistema fornece mensalmente boletim contendo os índices de acidentes com óbitos e com vítimas [InfoSiga 2016]. Também o Boletim de Acidente de Trânsito Eletrônico Unificado, (BATEU), do Estado do Paraná, viabiliza o registro de acidente de trânsito com apenas danos materiais, diretamente pelo cidadão na internet. Os relatórios estatísticos podem ser obtidos pela Polícia Rodoviária Estadual e o pelo Corpo de Bombeiros [BATEU, 2016]. Por fim, o Sistema de Declaração de Acidente de Trânsito, (DAT), registra via internet os casos de acidentes sem vítimas ocorridos nas rodovias

${ }^{1}$ COTRANS: http://www.inf.unioeste.br/ cotrans/

RIZZI, R. L.; RIZZI, C. B.; SILVA, I. F.; TOMINC, F. P.

Inovação em um Sistema de Informação para Gestão de Acidentes de Trânsito na Perspectiva de Produto e Processo

isys | Revista Brasileira de Sistemas de Informação, Rio de Janeiro, vol. 9, No. 3, pp. 05-25, 2016 
federais brasileiras. O cidadão acessa o site e preenche as informações requeridas. Esses dados são utilizados pela Polícia Rodoviária Federal [DAT 2016].

\subsection{SIGTRANS}

O Sistema Integrado Georreferenciado de Controle e Monitoramento de Acidentes de Trânsito (SIGETRANS) ${ }^{2}$ foi desenvolvido na Universidade Estadual do Oeste do Paraná em parceria com a Prefeitura Municipal de Cascavel por meio da Secretaria Municipal de Saúde. Realiza o gerenciamento de acidentes de trânsito com vítimas; de acidentes de trânsito sem vítimas; o acompanhamento hospitalar público e privado de acidentados de trânsito; o acompanhamento ambulatorial de acidentados de trânsito; o acesso ao Sistema (login e logout com acessos diferenciados). Também viabiliza a geração de relatórios e estatísticas; e ainda a visualização georreferenciada de eventos relacionados.

A Figura 1 ilustra a tela de inicial do Sistema que apresenta o mapa da cidade de Cascavel. Nela é possível identificar os acidentes com vítimas ocorridos no período de 15/05/2016 à 30/05/2016. Nessa tela, o usuário pode selecionar o período inicial e final que deseja consultar, e os fatores de análise, indicando se quer ou não que sejam apresentadas informações referentes aos acidentes com vítimas fatais, graves ou leves ou os acidentes sem vítimas. Viabiliza agrupar os acidentes por bairro ou por tipo de gravidade da ocorrência. A legenda apresentada à esquerda da Figura 1 ilustra os ícones referentes aos acidentes fatais, graves, leves e sem vítimas.

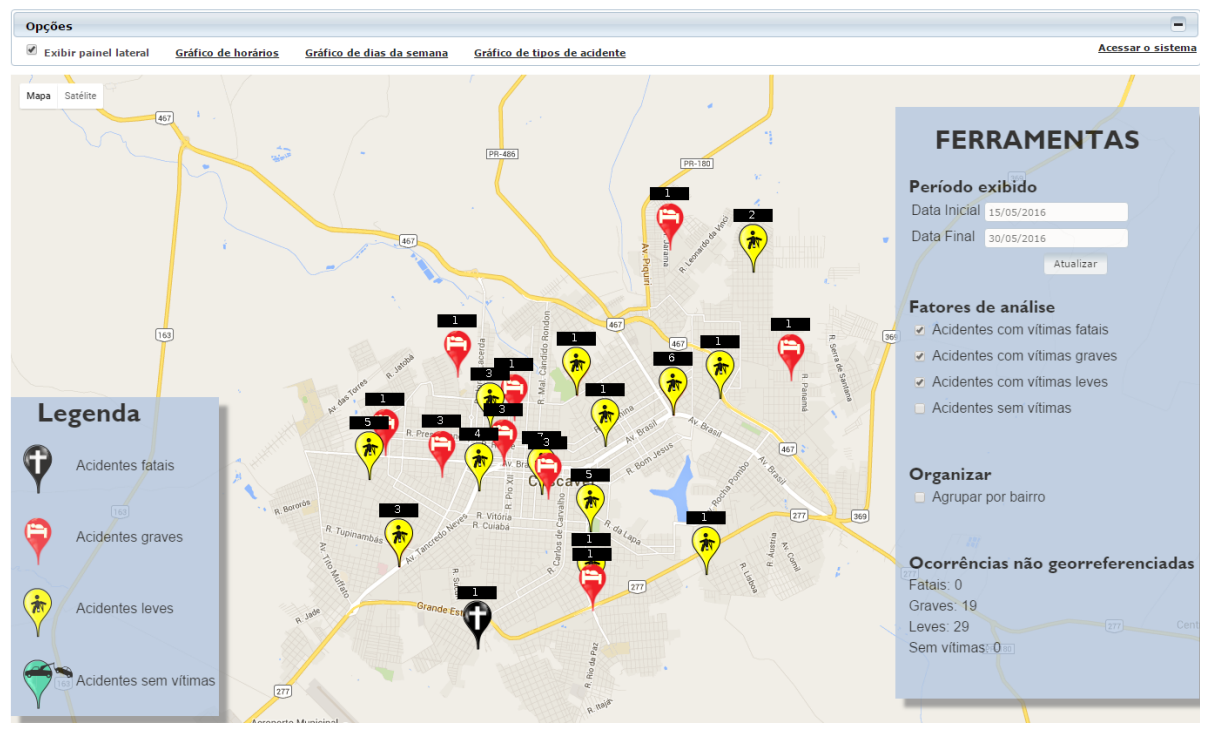

Figura 1: llustração da tela inicial do SIGETRANS apresentando o georreferenciamento dos acidentes com vítimas ocorridos em Cascavel no período 15/05/2016 à 30/05/2016.

Na parte superior direita da tela ilustrada na Figura 1, o usuário pode verificar os horários em que ocorrem os acidentes de trânsito no período solicitado. Pode identificar a quantidade de ocorrências agrupadas por dia da semana e os tipos de acidentes que ocorrem com mais frequência no período. Existe um controle de permissões, em que

${ }^{2}$ O Sistema SIGTRANS, nas primeiras versões, denominava-se de SIGETRANS. Pode ser acessado em http://sigetrans.cascavel.pr.gov.br:10081/sigetrans/. 
cada parceiro possui login e senha, e o usuário devidamente cadastrado deverá informálos para acessar as demais funcionalidades do SIGETRANS, ilustradas na Figura 2. Nela é possível identificar, no canto superior, operações relacionadas ao Sistema, e dentre elas destacam-se:

1. A visualização das ocorrências cadastradas manualmente pelos usuários naquele dia;

2. A inserção de Ocorrências como o Registro de Acidentes de Trânsito, RAS e o Boletim de Acidentes de Trânsito, BAT, que constituem os registros efetuados pelas instituições que realizam os primeiros socorros, como Corpo de Bombeiros e Polícia Rodoviária Estadual;

3. A inserção de informações sobre as vítimas atendidas pelas Unidades Básicas de Saúde, UBS, Clínicas, Unidades de Pronto Atendimento e Hospitais;

4. A consulta aos registros já cadastrados. É possível efetuar consultas específicas a determinadas vítimas, fornecendo seu nome ou pelo menos um dos seguintes itens, data de nascimento, nome da mãe, cartão SUS ou CPF;

5. A consulta de ocorrências registradas nos últimos 30 dias. Desta maneira, o usuário tem acesso à listagem por ordem de data. São exibidos além do código da ocorrência no Sistema, a data, o horário, o tipo do acidente e a rua onde os acidentes ocorreram. Essas informações viabilizam a busca da ocorrência que poderá ser consultada ou atualizada, selecionando a linha que representa o acidente de interesse;

6. Os relatórios estatísticos, como, por exemplo, a quantidade de acidentes de trânsito ocorrida por bairro, em um determinado período;

7. A visualização por gráficos que apoiam a execução da metodologia EPP;

8. O cadastramento de usuários no Sistema.

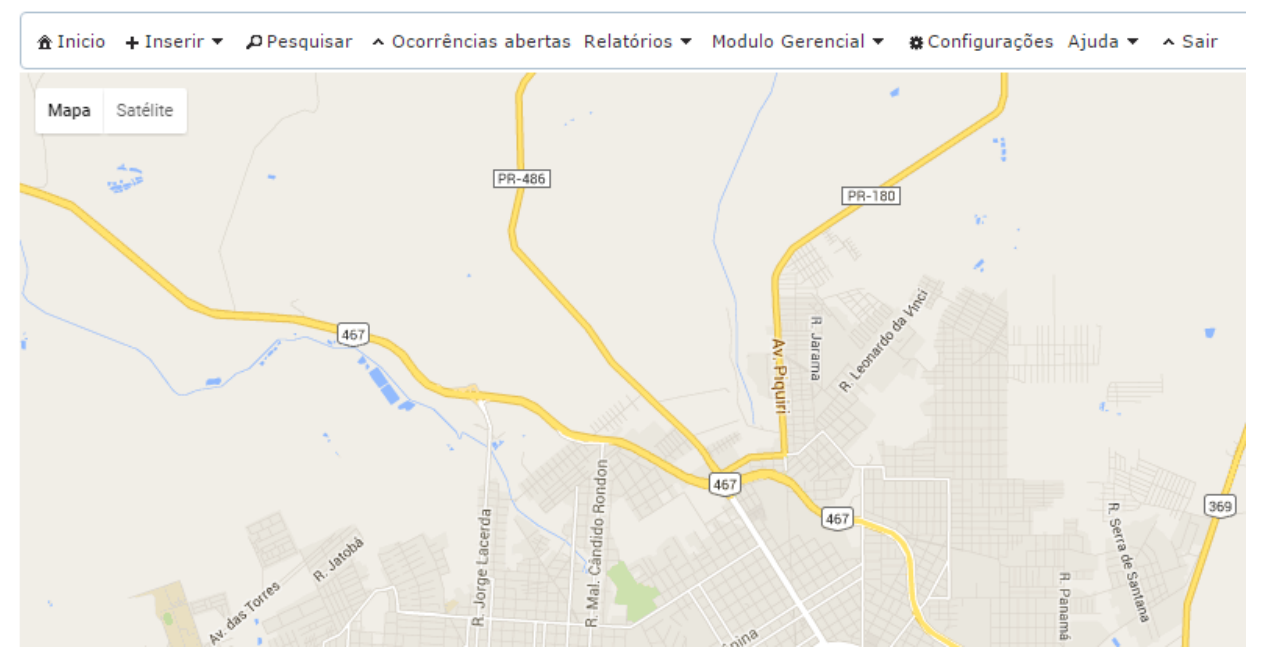

Figura 2: Tela interna do SIGETRANS com seu menu principal.

Desde a implantação do Sistema ocorrida em 2012, manutenções têm sido realizadas objetivando ajustá-lo às demandas dos usuários. Além dessas manutenções, novas demandas, especialmente em termos de soluções para evitar o retrabalho de digitação de dados já constantes nas bases de dados dos sistemas dos parceiros, motivou a equipe a evolui-lo, incluindo a revisão de seus requisitos. 
Optou-se por evoluir o Sistema com uma abordagem diferenciada na especificação e implementação do Sistema, voltada a microserviços [Newman 2015], que impactam diretamente na sua arquitetura. Consoante com essa concepção, a equipe optou também por substituir o paradigma de programação, migrando da programação imperativa à funcional, definindo, por consequência e necessidade, o uso de tecnologias distintas daquelas então utilizadas, e que atendessem às atuais necessidades.

Estas decisões justificaram-se não apenas a partir da experiência que a equipe de desenvolvimento teve com o SIGETRANS, mas com a insatisfação quanto à utilização de amplo e não integrado conjunto de tecnologias então utilizadas, que eram as disponíveis à época e de domínio pela equipe desenvolvedora. Para além das questões técnicas, as expectativas com relação ao desempenho da nova versão do ponto de vista do usuário também motivaram a construção da nova versão.

Essas questões são discutidas nas próximas seções, em que se realizam comparações entre a versão em uso e a em desenvolvimento. De modo geral, a nova versão contempla as principais funcionalidades apresentadas anteriormente, sendo ela designada Sistema de Informação e Gestão de Acidentes de Trânsito, SIGTRANS. Na sequência, são destacados aspectos de inovação tecnológica do SIGTRANS, quanto à sua concepção e às tecnologias que estão sendo utilizadas em seu desenvolvimento.

\section{Inovação Tecnológica no SIGTRANS}

Inovar significa tornar novo, renovar e, por conseguinte, inovação compreende o ato de inovar. Dentre as definições de inovação, uma das assumidas neste trabalho defende como inovação “... uma ideia, uma prática ou um artefato material percebido como novo, relevante e único adotado em um determinado processo, área ou por toda a organização." [Pereira 2016].

Embora esta concepção, inovação depende de sua aplicação. Quanto ao SIGTRANS é um software percebido como novo, relevante e único no contexto da gestão de acidentes e acidentados de trânsito, e neste sentido, trata-se de uma inovação do tipo disruptiva [Cândido 2011]. Ele ainda não chegou ao mercado para comercialização. No entanto, a partir de 2012, sua versão anterior, SIGETRANS, chegou ao usuário final, ou seja, está instalado na Prefeitura Municipal de Cascavel e está em uso por parceiros do município. Outra Prefeitura já manifestou interesse oficial, e tratativas estão sendo conduzidas no sentido de efetivar sua disponibilização.

Com relação à Inovação e à Pesquisa e Desenvolvimento Experimental no âmbito da indústria de Software, relatório técnico publicado recentemente discute os conceitos associados. Marco Aurélio Gerosa e colaboradores indicam que caracterizar o que é Inovação e Pesquisa e Desenvolvimento $(\mathrm{P} \& \mathrm{D})$ na área de software não é trivial [Gerosa et al 2016]. Citando Frescati [Frescati 2002], designam P\&D como:

\footnotetext{
Atividades que incluem o trabalho criativo empregado de forma sistemática com o objetivo de aumentar o corpo de conhecimento disponível e o uso desse corpo de conhecimento para conceber novas aplicações. Esse corpo pode incluir conhecimento sobre o homem, cultura e sociedade. Dessa definição, pode-se identificar alguns elementos-chave que toda $\mathrm{P} \& \mathrm{D}$ deve conter: o alvo da P\&D não é resolvível por meio da aplicação direta do que já se sabe (deve haver criação), a P\&D deve ser feita de forma sistemática (por
}

RIZZI, R. L.; RIZZI, C. B.; SILVA, I. F.; TOMINC, F. P.

Inovação em um Sistema de Informação para Gestão de Acidentes de Trânsito na Perspectiva de Produto e Processo

isys | Revista Brasileira de Sistemas de Informação, Rio de Janeiro, vol. 9, No. 3, pp. 05-25, 2016 
meio de processos e métodos bem definidos, como por exemplo os métodos científicos) e deve expandir o corpo de conhecimento existente e gerar novas aplicações. Não basta apenas uma dessas dimensões [Gerosa et al 2016], grifo deles.

Outro conceito importante diz respeito à definição de Desenvolvimento Experimental, e Gerosa e colaboradores o definem como:

O trabalho sistemático conduzido a partir de conhecimento originário de pesquisa e de experiências práticas, direcionado para produção de novos materiais, produtos ou dispositivos; para implantação de novos processos, sistemas e serviços; ou para melhorar substancialmente aqueles já produzidos ou implantados [Gerosa et al 2016].

Os autores destacam que este conceito pode contribuir para promover a concepção equivocada de que qualquer atividade no desenvolvimento de software pode ser considerada como Desenvolvimento Experimental. Atividades de inovação tecnológica objetivam levar à implementação de produtos ou processos novos ou melhorados do ponto de vista tecnológico. Porém, nem todas as atividades necessárias para implementar uma nova funcionalidade ou um novo produto constituem atividades de P\&D. São atividades de P\&D exclusivamente aquelas com risco tecnológico e com geração de conhecimento original [Gerosa et al 2016].

Assim, relativamente ao software, pode haver dúvidas na distinção entre a fase de $\mathrm{P} \& \mathrm{D}$, que geralmente envolve a geração de protótipos, e a transformação desses protótipos em produtos. Destaque-se que essa última fase, a de transformação do protótipo em produto, não se constitui $\mathrm{P} \& \mathrm{D}$. No entanto, é comum no desenvolvimento de software, que o protótipo torne-se parte do produto final. Assim, Gerosa e colaboradores entendem que a geração de protótipos fica no escopo da $\mathrm{P} \& \mathrm{D}$ enquanto ainda são necessárias modificações e testes para que o problema ou desafio tecnológico seja adequadamente superado.

Especificamente no desenvolvimento do Sistema houve etapas com atividades de P\&D que foram superadas quando de sua disponibilização à Prefeitura. Ocorre que como todo software, ele requereu manutenção e ajustes, que foram realizados à medida da necessidade. Mas, passados quatro anos de uso, a equipe de desenvolvimento optou por desenvolver nova versão, mais enxuta e moderna tecnicamente e enfatizando a maior automatização dos dados fornecidos pelos parceiros. Iniciou-se um trabalho de revisão e atualização de requisitos que culminou, inclusive com a mudança de sua denominação, passando a ser chamado de Sistema de Informação e Gestão de Acidentes de Trânsito (SIGTRANS). Assim, mais uma vez, atividades de P\&D estão continuamente sendo realizadas, em especial, quando os feedbacks chegam à equipe desenvolvedora.

Sobre outro aspecto, dentre as várias possibilidades de inovar, estão aquelas que se referem aos produtos ou processos que são ditas "inovações tecnológicas". De maneira abrangente, pode-se dizer, em concordância com o relatório de inovação inglês, que "inovação é a exploração com sucesso de novas ideias" [DTI 2003]. Além disso, as diretrizes para coleta e interpretação de dados sobre inovação, também denominado Manual do Oslo [OECD 2005], definem inovações em produtos ou processos como duas categorias de inovação, como: 
Uma inovação de produto é a introdução de um bem ou serviço novo ou significativamente melhorado no que concerne a suas características ou usos previstos. Incluem-se melhoramentos significativos em especificações técnicas, componentes e materiais, softwares incorporados, facilidade de uso ou outras características funcionais [OECD 2005 pg. 57].

Uma inovação de processo é a implementação de um método de produção ou distribuição novo ou significativamente melhorado. Incluem-se mudanças significativas em técnicas, equipamentos e/ou softwares [OECD 2005 pg. 58].

Neste contexto, a inovação para o Sistema de gestão de trânsito descrito neste artigo é discutida como combinação de inovações no produto e no processo de desenvolvimento do SIGTRANS. A inovação do produto ocorre por meio da incorporação em suas funcionalidades da metodologia EPP. A inovação do processo ocorre por meio do emprego de nova arquitetura do Sistema e do paradigma funcional, adotados para seu desenvolvimento que requereu mudança significativa nas técnicas adotadas.

Estas questões são discutidas na próxima seção, mas cabe reforçar que para as atividades de inovação relacionadas ao Sistema de gestão de trânsito relatado neste artigo, distintas atividades são realizadas. A primeira refere-se à Pesquisa e Desenvolvimento do Sistema, que incluiu o estudo da metodologia EPP; a identificação das funcionalidades do Sistema; o desenvolvimento e os testes de protótipos. A segunda diz respeito à Implantação do Sistema. Esta atividade inclui a aquisição de um repositório e serviços de aplicação de nuvem ${ }^{3}$, testes de aceitação do produto, incluindo feedbacks para melhoria, e treinamento no produto para sua implementação no ambiente real do usuário. Atividades relacionadas à preparação do mercado para as inovações do produto serão desenvolvidas de acordo com a disponibilização do SIGTRANS na nuvem, que ainda está em processo de desenvolvimento.

\subsection{Inovação no Produto - Metodologia e Funcionalidades}

Considerando que a inovação do produto pode estar baseada na combinação de conhecimentos existentes [OECD 2005], o SIGTRANS desde a sua versão anterior utiliza a metodologia EPP que é constituída por seis etapas [Cardita \& Di Pietro 2010].

- A Etapa 1 constitui a formação da parceria entre as entidades responsáveis pelo atendimento de ocorrências de trânsito, sendo de grande importância para o registro e integridade das informações pertinentes aos acidentes. No caso do Município de Cascavel, esta etapa foi contemplada pela formação COTRANS que reúne representantes da sociedade organizada e os responsáveis pelos atendimentos às vítimas e aos acidentes de trânsito no município [COTRANS 2016].

- A Etapa 2 constitui a coleta, gestão e análise dos dados para que medidas de prevenção e promoção da saúde e do trânsito sejam efetivamente adotadas. É necessária à formação de indicadores reais, fidedignos, atualizados e integrados. Esta etapa está sendo contemplada pelas atuais funcionalidades do SIGTRANS.

\footnotetext{
${ }^{3}$ A Amazon (https://aws.mazon.com) foi o serviço adquirido, pois atende os requisitos adotados no SIGTRANS.
} 
- A Etapa 3 constitui nas ações integradas de segurança viária, e propõe que sejam desencadeadas ações estratégicas baseadas no conhecimento obtido a partir dos dados coletados na etapa 2.

- A Etapa 4 constitui na monitoração de desempenho, avaliação e reconhecimento das estratégias adotadas mediante a redução dos índices de acidentes, vítimas fatais e feridos graves, definindo a eficiência das políticas de educação, prevenção e intervenção utilizadas com base nos dados obtidos nas etapas anteriores.

- A Etapa 5 constitui na revisão geral anual, e visa avaliar se os índices anuais apresentam reduções que atendem aos objetivos da EPP que são: a) dados confiáveis; b) redução de mortos e feridos graves; c) redução de custos com saúde; d) comunidades proativas para o trânsito seguro; e) melhoria da qualidade de vida.

- A Etapa 6 constitui na renovação e expansão, e visa à adaptação das estratégias às mudanças e inserção de novas parcerias tanto pelo setor público quanto privado.

Ressalta-se que as funcionalidades do SIGTRANS estão consistentes com a metodologia EPP, bem como, mantém as funções implementadas da versão anterior. Ele está estruturado considerando dois grandes enfoques, seus aspectos em termos de acidente e de acompanhamento às vítimas, conforme a modelagem conceitual ilustrada na Figura 3.
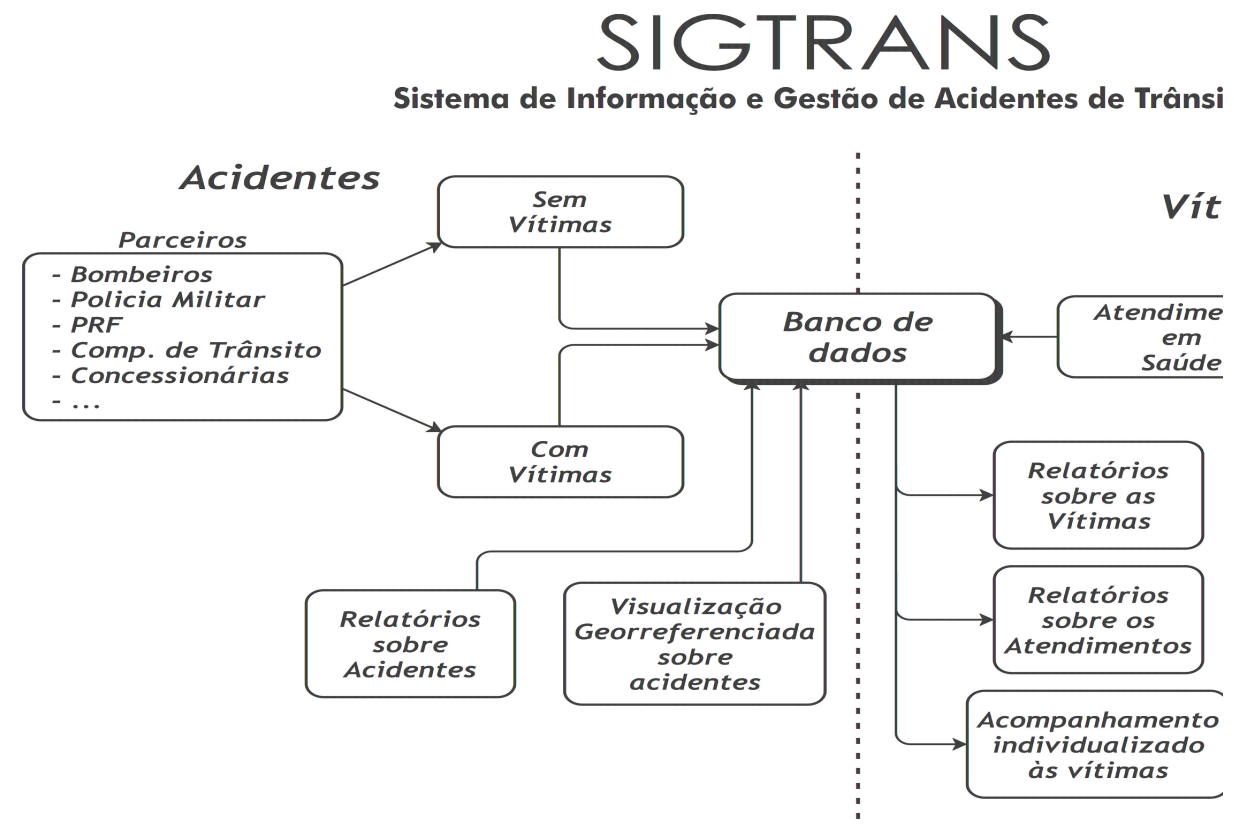

Figura 3. Modelagem conceitual de ambos os enfoques do SIGTRANS, os acidentes e as vítimas.

O Sistema viabiliza o registro e a identificação dos acidentes com e sem vítimas fornecidas pelos Parceiros. São Parceiros do SIGTRANS aquelas entidades públicas ou privadas que atuam no atendimento em decorrência de acidentes de trânsito, envolvido e vítimas, ocorridos no município e que fornecem os registros dos atendimentos realizados por eles para serem armazenados no Sistema, contribuindo para integração das informações. 
Como indicado na Figura 3, no que se refere ao primeiro enfoque, ao dos acidentes, os parceiros enviam arquivos no formato adequado contendo dados dos acidentes atendidos, ou os digitam diretamente no Sistema. Alternativamente, pode-se gerar uma planilha com os dados dos atendimentos gerados a partir do Sistema de Informação do parceiro, e inseri-lo diretamente no SIGTRANS por meio de funcionalidade específica. Atualizado o banco de dados do Sistema, viabiliza-se a geração de relatórios relativos aos acidentes, com e sem vítimas, assim como a visualização georreferenciada e a elaboração de mapas temáticos específicos.

No que se refere ao segundo enfoque, ao do acompanhamento às vítimas, os dados também podem ser fornecidos pelos parceiros por meio do envio de arquivo com a síntese dos atendimentos em saúde realizados para cada indivíduo. Eles são atualizados no banco de dados do SIGTRANS como um complemento aos dados do acidente, também viabilizando a geração de diversos relatórios relativos às vítimas, bem como a visualização georreferenciada do acidente.

Note-se que pode ocorrer situação em que a vítima é levada diretamente a uma instituição de saúde sem ter sido atendida por algum dos parceiros, bombeiros, polícia militar, entre outros, a exemplo do caso em que um ciclista é atropelado e conduzido ao hospital por populares. Nessa situação, não há o registro prévio do acidente, ocorrendo apenas o registro do atendimento em saúde em decorrência de um acidente. Também não há informações precisas a respeito do acidente em si, somente informação de que o indivíduo atendido foi vítima de acidente de trânsito. Mesmo constituindo um registro incompleto, entende-se que a informação é relevante ao SIGTRANS, visto que contribui para refletir melhor a realidade do trânsito no município.

Objetivando atender a estrutura desses enfoques, definiu-se a arquitetura do Sistema, tecnologias, paradigma de programação e ferramentas apropriadas para o processo de desenvolvimento do produto, que são discutidas a seguir.

\subsection{Inovação Quanto ao Paradigma de Programação, Arquitetura e Tecnologias}

Com o intuito de melhorar o desenvolvimento e manutenibilidade do SIGTRANS, mudanças no processo de construção e evolução do Sistema foram efetivadas. A equipe focou as mudanças no paradigma de programação e na arquitetura do software.

\subsubsection{Paradigma de Programação}

Do ponto de vista conceitual, pode-se dividir as linguagens de programação em quatro categorias: Imperativas, Funcionais, Lógicas e Orientadas a Objetos. Entretanto, como discutido por Robert W. Sebesta, geralmente não se considera que linguagens que suportam orientação a objetos formem uma categoria separada, visto que as extensões a uma linguagem necessárias para oferecer orientação a objetos não são tão complexas, sendo então, um conceito ortogonal aos demais [Sebesta 2000].

Programação funcional recebe essa designação devido ao fato de que sua operação fundamental é a aplicação de funções aos argumentos. O próprio programa principal é escrito como uma função que recebe a entrada do programa como seu argumento e retorna a saída do programa como resultado. Tais funções derivam de funções matemáticas, com as mesmas propriedades [Hughes 1990]. 
O paradigma funcional não é adotado pela maioria dos desenvolvedores de sistemas de informação, os quais preferem o paradigma imperativo. Os motivos podem ser variados, mas a complexidade de troca de paradigmas e a ideia de que o paradigma funcional não seria adequado para sistemas de informação afetam a decisão quanto à sua adoção [WADLER 1998].

Optou-se por utilizar este paradigma para o desenvolvimento da nova versão do Sistema, dentre outros aspectos, porque a programação funcional contribui para que o programador desenvolva as funcionalidades com menor quantidade de erros [Ray et. al 2014], maior modularidade [Hughes 1990], e menor complexidade na implementação concorrente do mesmo [Lutz 2013] impactando na manutenibilidade e corretude do software, contribuindo na qualidade do produto final.

\subsubsection{Microserviços e a Atual Arquitetura do SIGTRANS}

Software como microserviços são pequenos serviços autônomos que trabalham em conjunto [Newman 2015], sendo que as aplicações de software são modularizadas em pequenas partes - os microserviços - em que cada uma delas realiza sua funcionalidade com alto grau de coesão.

Conceitualmente, microserviços emergem de relevantes trabalhos à melhoria do desenvolvimento de software, a exemplo do Desenvolvimento Dirigido ao Domínio (DDD) [Evans 2003], Entrega Contínua [Humble e Farley 2010] e Sistemas Reativos [The Reactive Manifesto 2016]. Por meio de DDD, a decomposição funcional é endereçada por microserviços. A Entrega contínua contribui para que cada microserviço possa ser distribuído, atualizado, substituído e escalado de maneira ágil. O Sistema Reativo fornece quatro princípios, responsividade, elasticidade, resiliência e dirigido à mensagem, para o projeto de software flexível, com baixo acoplamento e escalável.

Por conseguinte, adotar microserviços no desenvolvimento de software fornece benefícios, dentre eles a independência no dimensionamento, distribuição, manutenção, e escolha da linguagem de desenvolvimento. Essa independência é alcançada por meio de características que eles possuem. A característica da autonomia, por exemplo, viabiliza que um microserviço seja uma entidade separada e assim, ele pode ser distribuído como uma parte do software isolada sobre uma plataforma [Newman 2015]. A característica da resiliência viabiliza que o sistema permaneça em execução, ainda que um microserviço falhe.

No contexto do SIGTRANS, microserviços promovem inovação de duas maneiras, por meio dos objetivos estratégicos para o negócio e das características arquiteturais para o desenvolvimento do sistema. No que tange aos objetivos estratégicos, microserviços habilita escalar os negócios da organização, em que mais clientes/transações são habilitados, assim como, a ideia de self-service para o cliente. Além do mais, há suporte para a entrada em novos mercados por meio de processos operacionais mais flexíveis. O SIGTRANS objetiva escalar ao atender diversas prefeituras, ou demais organizações com interesse em trânsito, assim como ter flexibilidade por meio da rápida manutenção e evolução dos microserviços.

Essa inovação estratégica é alcançada pelas características subjacentes da arquitetura de microserviço. Além da autonomia e resiliência, há responsividade, 
situação em que o sistema deve responder em tempo hábil, a elasticidade, em que é possível de se escalar o sistema tanto verticalmente quanto horizontalmente sob carga de trabalho variável, e a característica de ser dirigido à mensagem, no qual o sistema é baseado na passagem de mensagens de maneira síncrona ou assíncrona.

A estrutura exibida na Figura 3 contém vários requisitos que definem os microserviços da Figura 4, que mostra a arquitetura do SIGTRANS. Nela, os microserviços Relatórios, Ocorrências, Acompanhamento Hospitalar e Ocorrências Abertas estão isolados entre si, porém, eles podem se comunicar. Cada microserviço possui exclusivamente sua base de dados, em que se emprega REST (Representational State Transfer), um estilo de arquitetura de software para sistemas distribuídos [Fielding 2000] para realizar a interface entre o front-end e o back-end do sistema. Além do mais, se emprega uma API Gateway, que é um servidor HTTP em que rotas são configuradas e associadas a cada microserviço.

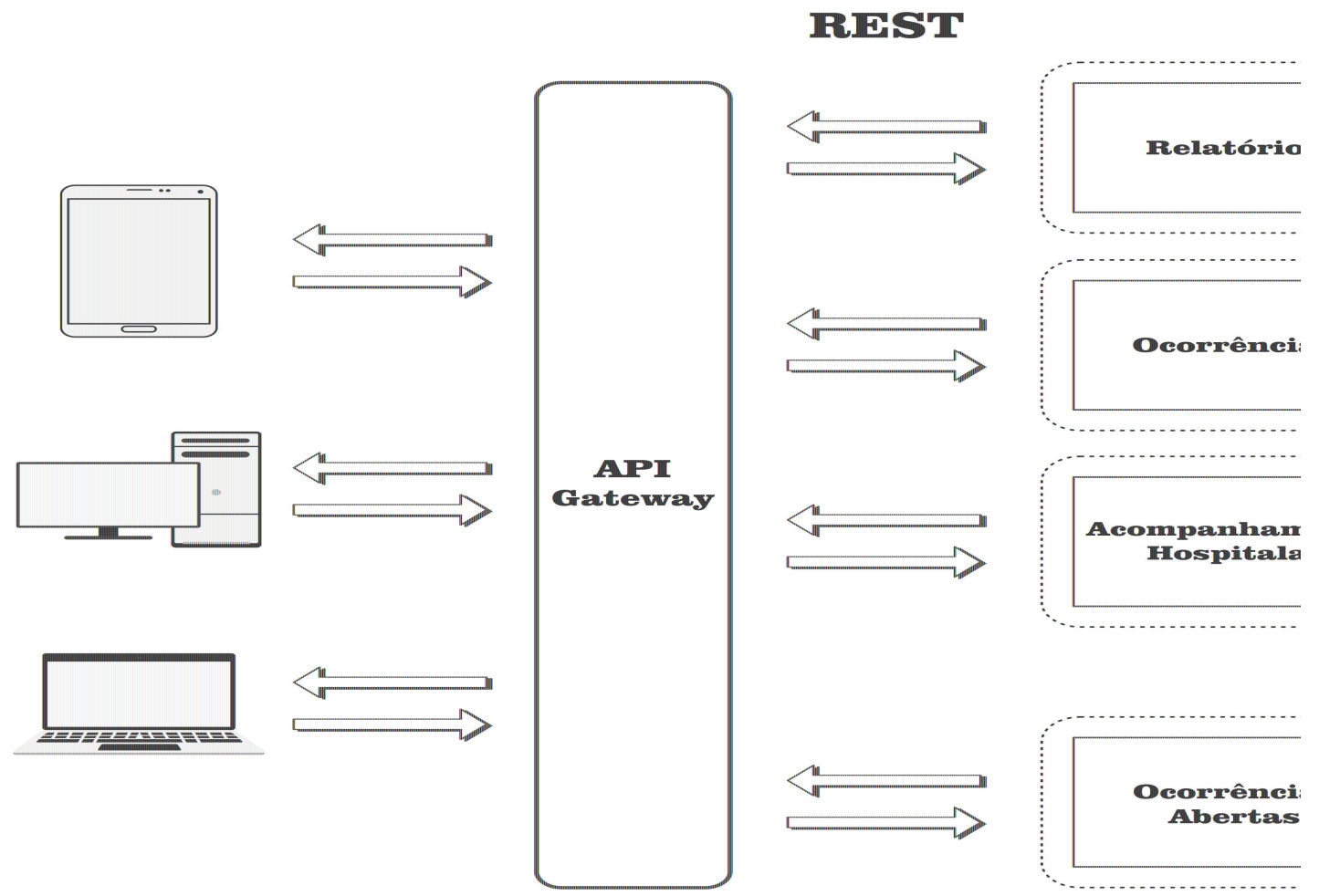

Figura 4: Arquitetura de microserviços do SIGTRANS.

\subsubsection{Tecnologias Utilizadas no SIGETRANS e no SIGTRANS}

No desenvolvimento da versão anterior do SIGTRANS, o SIGETRANS, foram utilizadas as ferramentas listadas na Tabela 1. Como a arquitetura daquela versão é monolítica todos os artefatos de software foram produzidos assim baseados.

Tabela 1: Ferramentas utilizadas para o desenvolvimento do SIGETRANS.

\begin{tabular}{|l|ll|}
\hline Ferramenta & Finalidade \\
\hline PostgreSQL [2016] & Sistema gerenciador de banco de dados relacionais. \\
\hline PostGis [2016] & Extensão do PostgreSQL para uso de georreferenciamento. \\
\hline Hibernate [2016] & Framework utilizado para mapeamento objeto-relacional. \\
\hline Hib.Spatial [2016] & Extensão do Hibernate para objetos geométricos e \\
\hline
\end{tabular}




\begin{tabular}{|l|l|}
\hline & georreferenciados. \\
\hline Spring [2016] & Framework para desenvolvimento de aplicações Java em camadas. \\
\hline Spring Security [2016] & Realiza a autenticação e autorização de usuários. \\
\hline JSF [2016] & Utilizado para construção da visão do Sistema. \\
\hline Primefaces [2016] & Biblioteca de componentes gráficos para JSF. \\
\hline Glassfish [2016] & Servidor de aplicação. \\
\hline Google Maps [2016] & API utilizada para georreferenciamento de endereços. \\
\hline Maven [2016] & Viabiliza referenciar dependências do projeto. \\
\hline Subversion [2016] & Viabiliza o controle de versão e alteração de código. \\
\hline Netbeans [2016] & IDE utilizada. \\
\hline iReport [2016] & Utilizada para construir relatórios. \\
\hline Java [2016] & Linguagem de programação Imperativa \\
\hline
\end{tabular}

A Figura 5 mostra o diagrama de classes das Ocorrências no SIGETRANS destacando as interdependências das classes envolvidas, o que contrasta com o diagrama do microserviço equivalente no SIGTRANS, como apresentado na Figura 6.

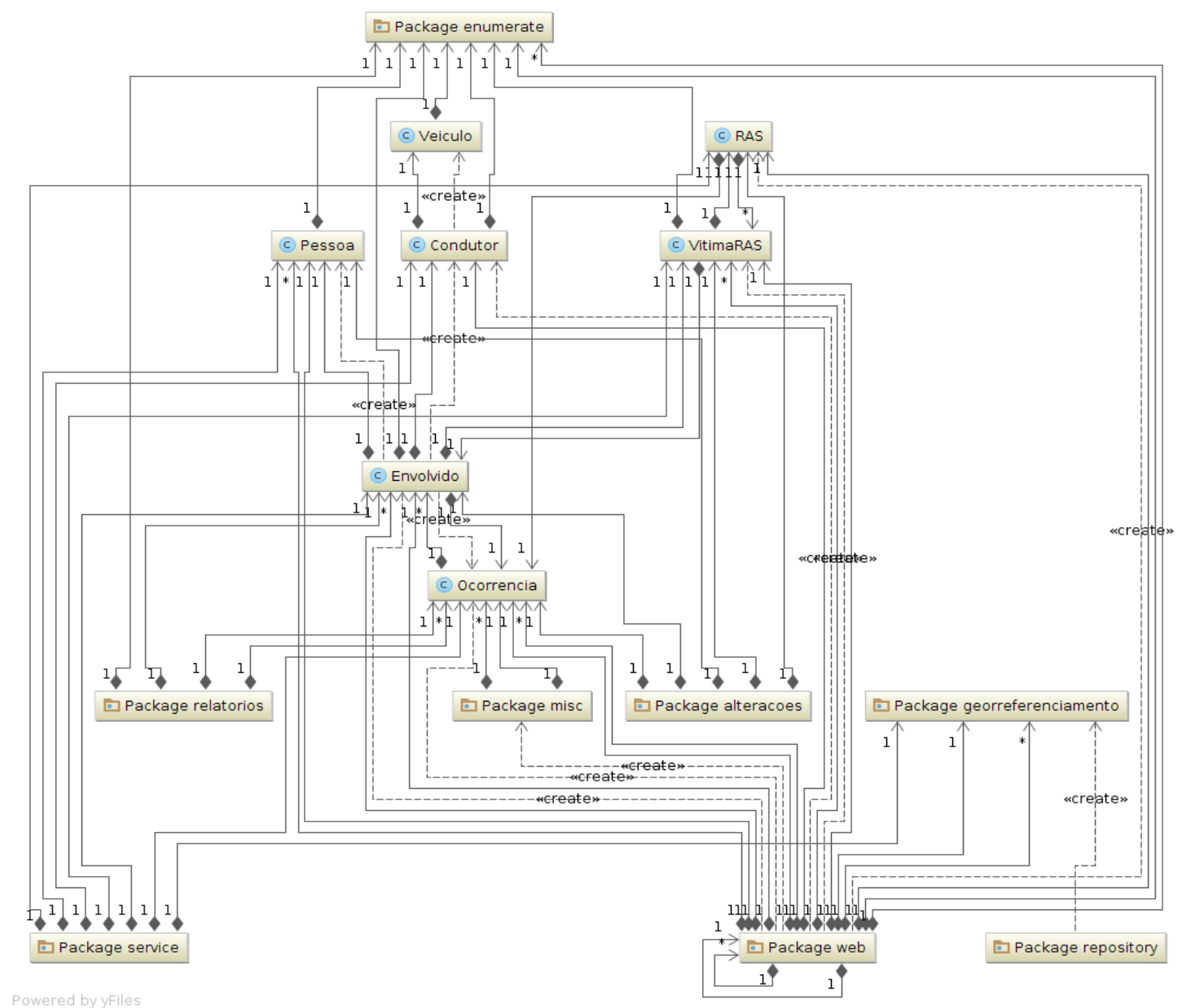

Figura 5: Diagrama de Classes de Ocorrências no SIGETRANS.

Por sua vez, no desenvolvimento do Sistema SIGTRANS, que utiliza a arquitetura de microserviços, estão sendo utilizadas as ferramentas listadas na Tabela 2.

Tabela 2: Ferramentas utilizadas para o desenvolvimento do SIGTRANS 


\begin{tabular}{|l|l|}
\hline Ferramenta & Finalidade \\
\hline Intelij Idea [2016] & $\begin{array}{l}\text { Ambiente de desenvolvimento integrado para desenvolvedores de } \\
\text { software. }\end{array}$ \\
\hline PostgreSQL [2016] & Sistema gerenciador de banco de dados relacionais. \\
\hline Spray Toolkit [2016] & $\begin{array}{l}\text { Open source voltado à construção de camadas de integração baseada } \\
\text { no REST/HTTP sobre Scala e Akka. }\end{array}$ \\
\hline Scala [2016] & Linguagem de programação funcional e Orientada a Objetos. \\
\hline Git [2016] & Sistema de controle de versões distribuído. \\
\hline Github [2016] & Website para armazenamento de repositórios Git na nuvem. \\
\hline JSON [2016] & $\begin{array}{l}\text { JavaScript Object Notation, padrão para transmissão e } \\
\text { armazenamento de informações. }\end{array}$ \\
\hline Google Maps [2016] & API utilizada para georreferenciamento de endereços. \\
\hline Akka [2016] & $\begin{array}{l}\text { Toolkit e runtime para desenvolvimento de aplicações altamente } \\
\text { concorrentes, distribuídas e resilientes sobre JVM [2016]. }\end{array}$ \\
\hline SBT [2016] & Scala Build Tool. \\
\hline Slick [2016] & Biblioteca de acesso e consulta a banco de dados para Scala. \\
\hline
\end{tabular}

No SIGTRANS cada microserviço possui sua estrutura de classes, banco de dados, e demais elementos necessários para seu funcionamento. A Figura 6 mostra um exemplo para o diagrama de Classes do microserviço Ocorrências.

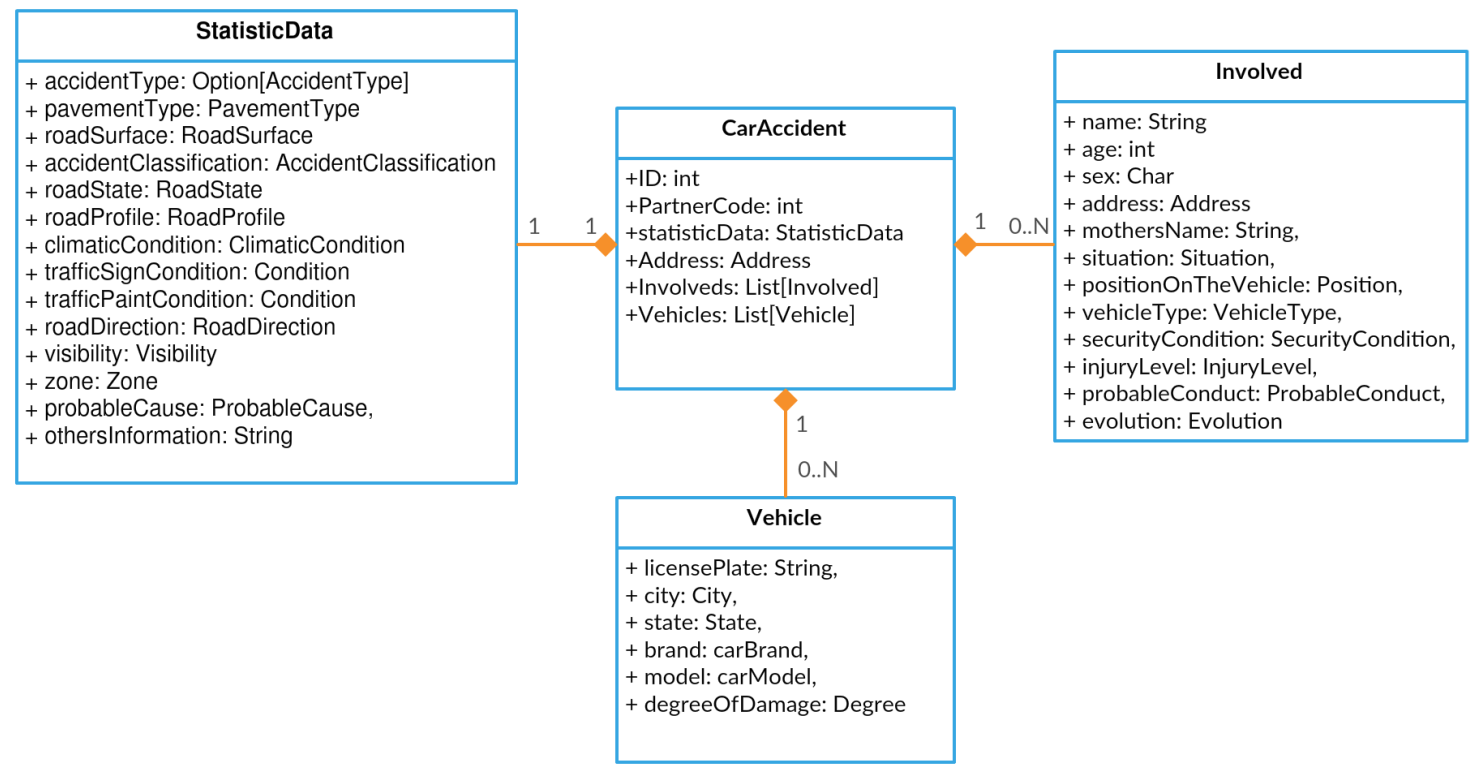

Figura 6: Diagrama de classes no SIGTRANS - Microserviço Ocorrências.

Uma comparação entre as Tabelas 1 e 2 indica que as ferramentas utilizadas em ambos os Sistemas são PostgreSQL e Google Maps. A distinção entre as tecnologias utilizadas no SIGETRANS e SIGTRANS reflete as diferenças entre as abordagens empregadas, uma monolítica e outra baseada em serviços. As ferramentas Intelij Idea, Git/GitHub substituem Netbeans e Subversion, e independem da mudança arquitetural. Já as ferramentas Spray Toolkit, Scala, JSON, SBT, Slick e Akka foram selecionadas por facilitar a implementação do paradigma funcional (Scala) e da arquitetura de microserviços. Scala substitui a linguagem Java e $S B T$ substitui a ferramenta Maven. As demais ferramentas tais como JSF e Hibernate referem-se ao mundo Java de desenvolvimento. 


\subsection{Lições Aprendidas, Limitações e Desafios Futuros}

A seguir, destacam-se lições aprendidas e limitações a partir do desenvolvimento do SIGTRANS, bem como, os impactos identificados no processo e no Sistema.

\subsubsection{Metodologia e Funcionalidades no SIGTRANS}

Como mencionado na seção 2, os municípios enfrentam um sério problema com o registro dos acidentes de trânsito ocorridos, visto que eles são armazenados em bases de dados de várias entidades, a exemplo do Corpo de Bombeiros, Polícia Militar, Entidades de Saúde e outros órgãos, mas esses registros nem sempre estão completos ou corretos. Também, da fragmentação decorrente do armazenamento em meios heterogêneos, como formulários impressos, tabelas e planilhas eletrônicas ou em sistemas computacionais com bases de dados diferentes e não relacionadas, decorrem estatísticas imprecisas que não refletem a realidade do problema.

O que os municípios geralmente fazem é solicitar mês a mês que cada entidade que armazena informações sobre acidentes de trânsito forneça as informações de que dispõe para fins de cômputo geral realizado pela equipe de vigilância epidemiológica. Esse processo é manual, lento, burocrático e geralmente não viabiliza registros rápidos e fidedignos. Objetivando superar essa problemática com o apoio institucional do COTRANS foi adotada a metodologia EPP divulgada pelo Global Road Safety Partnership que define por meio de suas seis etapas a sistemática de como bem conduzir as ações em termos de trânsito e suas vítimas. Assim, quando da concepção do SIGTRANS, ele foi estruturado objetivando contemplar essa metodologia, por meio de suas funcionalidades.

O SIGTRANS, com os dados que disponibiliza, contempla a etapa 2 que constitui a coleta, gestão e análise dos dados, subsidiando medidas de prevenção e promoção da saúde e do trânsito por meio de indicadores reais e atualizados. Os relatórios e mapas georreferenciados que dispõem, viabiliza que a etapa 3 seja realizada, ou seja, o desencadeamento de ações baseadas nos dados efetivos. As ações no âmbito do município atingiram até o momento a etapa 3. As demais, 4, 5 e 6, ainda precisam ser efetivamente implementadas com ações práticas que dependem não apenas de vontade política, mas de recursos financeiros e de pessoal. Quando essas etapas forem sendo realizadas e atingidas o SIGTRANS as apoiará com informações, relatórios específicos e visualização em mapas temáticos.

\subsubsection{Arquitetura e Tecnologias Adotadas no Desenvolvimento do SIGTRANS}

As mudanças são radicais no que se refere à arquitetura, tecnologia e a curva de aprendizado. Em especial, nota-se um impacto no esforço para migrar a arquitetura monolítica para a de microserviços. Toda a estrutura da aplicação está sendo refatorada para pequenos serviços, os quais possuem autonomia por meio de suas regras de negócio e base de dados embutidas e autossuficientes. Reestruturar esta arquitetura exige a construção de cada microserviço com essas características.

A estrutura do domínio da aplicação - Sistemas de gestão de acidentes de trânsito - motivou as alterações na estrutura e no processo de desenvolvimento do Sistema. A adoção da tecnologia de microserviço é resultado da análise dessa estrutura. 
Um benefício menos óbvio, mas relevante, está relacionado à demanda por microserviços por parte dos consumidores a um conjunto de interfaces do microserviço que os projetistas não haviam imaginado durante seu desenvolvimento. Esta concepção contrasta com a abordagem monolítica, em que um stakeholder deve ser consultado antes de uma integração. Como impacto deste benefício, destacam-se a manutenibilidade e evolução SIGTRANS, pois os projetistas dos microserviços poderão tomar decisões rapidamente sobre parte do Sistema sem que se afete o todo.

A linguagem funcional adotada, Scala, contribui para melhoria na qualidade do código [Ray et al 2014], o que avaliza a decisão inovadora de mudança de paradigma no projeto. Mudança como esta não é convencional em projetos de sistemas de informação.

Contudo, o desenvolvimento de software usando paradigma funcional requer esforço até que os programadores tenham maturidade, sobretudo quando eles estão mais familiarizados com linguagens imperativas. E como principal lição aprendida neste cenário, a equipe SIGTRANS está adotando o paradigma funcional de maneira incremental, ou seja, o SIGTRANS está sendo evoluído utilizando também o paradigma imperativo. O que parece ser uma limitação, essa evolução do SIGTRANS remete para uma mudança incremental e sem traumas para a equipe.

\subsubsection{Inovação no SIGTRANS}

A inovação no SIGTRANS pode ser identificada e implementada pela combinação de pequenas mudanças no produto, no processo, na metodologia, nas tecnologias e em outras categorias de inovações. Se compararmos com outros trabalhos relacionados a este, o conjunto de mudanças integradas no Sistema SIGTRANS sintetiza a inovação para gestão de acidentes de trânsito fornecendo além do emprego das funcionalidades e metodologia, uma arquitetura escalável para o crescimento do uso do Sistema, sustentado em tecnologias modernas viabilizando aos desenvolvedores maior manutenibilidade da aplicação em futuras atualizações.

O Projeto que sustenta o SIGTRANS tem como estratégia de inovação a adoção do conjunto melhorias concretizadas no produto (metodologia e funcionalidades) e no processo (arquitetura e paradigma de programação) para desenvolvimento do produto. Um desafio que está sendo enfrentado pela equipe SIGTRANS diante dessas mudanças tecnológicas e arquitetural é o esforço de aprendizado, já que essas tecnologias não são trabalhadas em cursos tradicionais na área de tecnologia da informação. Para atenuar os riscos envolvidos com este desafio, são ministrados treinamentos sobre as tecnologias adotadas para os membros ligados ao projeto.

\section{Considerações Finais}

Parte significativa do tempo das atividades humanas é destinada à locomoção, realizada em maior escala por meio de transporte terrestre. O aumento substancial da frota e fluxo de veículos, o sistema de transporte de massa insuficiente e ineficiente e as necessidades crescentes de movimentações de indivíduos e de cargas, dentre outros fatores, integram a dinâmica do trânsito e contribuem para que os acidentes aconteçam.

O SIGTRANS é um Sistema de Informação Geográfica que viabiliza análises com formação e alternação de cenários, numa perspectiva georreferenciada, facilitando a tomada de decisões, contribuindo para a melhor compreensão dos problemas 
relacionados aos acidentes de trânsito. Ele visa, dentre outros aspectos, apoiar a gestão municipal oferecendo informações para que especialistas e não especialistas das áreas de trânsito, saúde pública, corpo de bombeiros, dentre outros interessados, possam analisálas com dimensão espacial e temporal, relacionando-as para tomada de decisão fundamentada em dados.

O Sistema fornece informações confiáveis e com alta disponibilidade, viabilizando, entre outras características, acesso e manipulação de dados como os constantes nos registros de acidentes com vítimas, que expressam quantitativa e qualitativamente a severidade e agravos da ocorrência. É extensível a um ou mais municípios desde que os dados estejam disponíveis e as parcerias necessárias estejam estabelecidas.

As principais inovações do SIGTRANS estão categorizadas como inovações de produto e de processo. A inovação de produto está atrelada a adoção da metodologia EPP e de funcionalidades apropriadas para um sistema de gestão de acidentes e acidentados. Essas características distinguem o produto de outras soluções computacionais no mesmo segmento de mercado.

A inovação do processo está atrelada ao uso de microserviços e da programação funcional por meio da linguagem Scala, os quais forneceram uma arquitetura de software que suportava as funcionalidades do Sistema em uma codificação com menor propensão a erros de programação. Em especial a arquitetura de microserviço viabiliza escalar o atendimento para várias prefeituras, com processos operacionais mais flexíveis, autonomia e possibilidade de inovação por parte dos consumidores dos microserviços.

Como exemplo de dificuldades no desenvolvimento do SIGTRANS cita-se o esforço para compreensão do código no paradigma funcional e o refatoramento da arquitetura antiga (monolítica) para microserviços. Esse esforço implica em tempo extra para entendimento, alteração e testes do Sistema.

Estão em andamento estudos comparativos entre os paradigmas de programação imperativo por meio da linguagem Java, e o funcional por meio da linguagem Scala, considerando funcionalidades ou microserviços, como aqueles discutidos na seção 3.2.3 relativos às Ocorrências. Nesses estudos será empregada a metodologia de experimentação controlada, [Basili et al 1986] para realização de estudos sistemáticos no contexto de confiabilidade e corretude do código.

O projeto que alicerça o Sistema SIGTRANS está atuando para expandir seu uso por usuários de outros municípios. Essa estratégia de negócio, que pretende escalar o uso do software por meio dos microserviços, utilizará os serviços externos de computação em nuvem e outras prefeituras poderão utilizar a aplicação. E outro estudo sobre a escalabilidade por meio dos microserviços poderá ser realizado com o propósito de analisar atributos de qualidade como o desempenho e a segurança da aplicação.

\section{Agradecimentos}

Agradecemos à Fundação Araucária, ao CNPq e à Prefeitura Municipal de Cascavel pelo financiamento parcial ao projeto. À Unioeste pela logística. Aos parceiros pela colaboração tanto no desenvolvimento quanto na utilização do Sistema. 
Os autores também agradecem aos revisores que por meio de sugestões e comentários, contribuíram para o aprimoramento do trabalho.

\section{Referências}

Akka (2016). http://akka.io/

Babtie Group Ltda. (1999). Urban Traffic Management and Control (UTMC). http://tap.iht.org/objects_store/199910/IHT\%20NMN\%20Urban\%20Traffic \%20Management $\% 20$ and $\% 20$ Control $\% 20$ Systems.pdf

Basili, V. R. and Selby, R. and Hutchens, D. (1986). Experimentation in software engineering. IEEE Transactions on Software Engineering.

Brasil. (2009). Governo Federal. Década de ação pela segurança no trânsito - 20112020. Proposta para o Brasil para redução de acidentes e segurança viária. http://www.denatran.gov.br/download/decada/Proposta\%20ANTP-CEDATTInstituto $\% 20 \mathrm{de} \% 20$ Engenharia $\% 20$ SP.pdf.

Cândido. A. C. (2011). Inovação Disruptiva: Reflexões sobre as suas características e implicações no mercado. IET Working Papers Series No. WPS05/2011. https://run.unl.pt/bitstream/10362/6912/1/WPSeries_05 2011ACC\%C3\%A2ndido$1 . \mathrm{pdf}$

Cardita, J. and Di Pietro, G. (2010). PPS, Proactive Partnership Strategy. A Community Participation Model to address Road Safety. http://grsp.drupalgardens.com/sites/grsp.drupalgardens.com/files/GRSP-PPSmanual-290310.pdf

Cotton, B. (2015). Frost \& Sullivan. Intelligent Urban Transportation Predicting, Managing, and Integrating Traffic Operations in Smarter Cities. http://www935.ibm.com/services/multimedia/Intelligent_Urban_Transportation.pdf

Dti. (2003). Innovation Report. http://webarchive.nationalarchives.gov.uk/ +/http:/www.dti.gov.uk/files/file12093.pdf

Evans, E. (2003). Domain-Driven Design: Tackling Complexity in the Heart of Software. $1^{\circ}$. Edition. Addison-Wesley Professional.

Fielding, T. T. (2000). Architectural Styles and the Design of Network-based Software Architectures. Ph.D. Thesis. University of California, Irvine. Information and Computer Science. https://www.ics.uci.edu/ fielding/pubs/dissertation/fielding dissertation.pdf

Frescati, M. (2002). Méthode type proposée pour les enquêtes sur la recherche et le développement experimental. http://www.stis.belspo.be/docs/pdf/Frascati2002 finalversion_f.pdf.

Gerosa M. A. et al. (2016). O que é Inovação e P\&D na Indústria de Software? RTMAC-2016-01. https://www.ime.usp.br/ gerosa/inovacao/InovacaoEmSoftware-RTMAC-2016-01.pdf

Git (2016). https://git-scm.com/

Github (2016). https://github.com/ 
Glassfish (2016). https://glassfish.java.net/

Google Maps API (2016). https://developers.google.com/maps/?hl=pt-br

Grsp, Global Road Safety Partnership http://www.grsproadsafety.org/partners/become.

Hibernate (2016). http://hibernate.org/

Hibernate Spatial (2016). http://www.hibernatespatial.org/

Hughes, J. (1990). Why Functional Programming Matters. Research Topics in Functional Programming. D. Turner, Addison-Wesley, pp 17-42.

Humble, J. and Farley, D. (2010). Continuous Delivery. $1^{\circ}$. Edition. Addison-Wesley Professional.

Intelij Idea (2016). https://www.jetbrains.com/idea/.

Ipea. (2006). Impactos sociais e econômicos dos acidentes de trânsito nas rodovias brasileiras - Relatório Final. Brasília: IPEA/DENATRAN/ANTP. 242 p.

Ipea. (2015). Acidentes de Trânsito nas Rodovias Federais Brasileiras - Caracterização, Tendências e Custos para a Sociedade. Relatório de Pesquisa. Brasília: IPEA. 42p.

Ipeaa. (2006). Impactos sociais e econômicos dos acidentes de trânsito nas rodovias brasileiras - Relatório Executivo. Brasília: IPEA/DENATRAN/ANTP. 80 p.

iReport (2016). http://community.jaspersoft.com/project/ireport-designer

Java (2016). https://www.java.com/pt BR/

Java Virtual Machine

http://docs.oracle.com/javase/specs/jvms/se8/html/index.html

(2016).

JSF (2016). https://javaserverfaces.java.net/

JSON (2016). http://www.json.org/

Log4J (2016). http://logging.apache.org/log $4 \mathrm{j} / 2 . \mathrm{x} /$

Lutz M. J. (2013), "Functional approaches to teaching concurrency," 2013 IEEE Frontiers in Education Conference (FIE), Oklahoma City, OK, 2013, pp. 432-434. doi: 10.1109/FIE.2013.6684861

Maven (2016). https://maven.apache.org/

Mesquita Filho, M. (2012). Acidentes de trânsito: as consequências visíveis e invisíveis à saúde da população. Revista Espaço Acadêmico. V. 11, n. 128.

Netbeans (2016). https://netbeans.org/

Newman, S. (2015). Building Microservices: Designing fine-grained Systems. 1. Edition. O'Reilly Media.

OECD/Eurostat. (2005). Oslo Manual: Guidelines for Collecting and Interpreting Innovation Data, 3rd Edition, OECD Publishing, Paris.

Pereira, R. (2016). Os conceitos de inovação. http://www.pr2.ufrj.br/programas/programas/inovacao/attach/14-01.pdf

RIZZI, R. L.; RIZZI, C. B.; SILVA, I. F.; TOMINC, F. P.

Inovação em um Sistema de Informação para Gestão de Acidentes de Trânsito na Perspectiva de Produto

iSys | Revista Brasileira de Sistemas de Informação, Rio de Janeiro, vol. 9, No. 3, pp. $05-25,2016$ 
Por Vias Seguras. Estatísticas nacionais de acidentes de trânsito. (2016) http://www.vias-seguras.com/os_acidentes/estatisticas/estatisticas_nacionais

PostGis (2016). http://postgis.net/

PostgreSQL (2016). https://www.postgresql.org/

Primefaces (2016). http://primefaces.org/

Ray, B. and Posnett, D. and Filkov, V. and Devanbu, P. T. (2014). A Large Scale Study of Programming Languages and Code Quality in Github. Proceedings of the 22nd ACM SIGSOFT International Symposium on Foundations of Software Engineering, Pg. 155-165

SBT (2016). http://www.scala-sbt.org/index.html

Scala (2016). http://www.scala-lang.org/

SCOOT. (2012). Scoot user guide. Report 666/HF/16940/000. Siemens Mobility, Traffic Solutions. System/Project/Product: Siemens Mobility, Traffic Solutions UTC. http://www.siemens.co.uk/traffic/pool/downloads/handbooks/utc/666_hf_16940_000 .pdf

Sebesta, R. W. (2000). Conceitos de Linguagens de Programação. $4^{\circ}$ edição: Porto Alegre, Bookman, 2000.

Slick. (2016). http://slick.lightbend.com/

Souza. R. S. L. Os Acidentes de Trânsito e a Responsabilidade Civil do Estado. (2016). http://www.oabcampos.org.br/artigo.php?id=17

Spray. (2016). http://spray.io/

Spring Security. (2016). http://projects.spring.io/spring-security/

Spring. (2016). https://spring.io/

Subversion. (2016). https://subversion.apache.org/

The Reactive Manifesto. (2016). http://www.reactivemanifesto.org/

Transitobr. (2016) O portal do trânsito brasileiro. Acidentes - Causas. http://www.transitobr.com.br/index2.php?id_conteudo $=8$

Wadler, Philip. (1998). Why no one uses functional programming languages. ACM Sigplan Notices, New York, NY, US, p. 23-27. 